\title{
Cooperación entre las Familias y los Centros Escolares como Medida Preventiva del Fracaso y del Riesgo de Abandono Escolar en Adolescentes
}

\author{
Family-School Partnerships as a Measure to Prevent School \\ Failure and the Risk of Dropping Out in Adolescents
}

\author{
Lucía Álvarez Blanco * \\ Raquel-Amaya Martínez-González \\ Universidad de Oviedo
}

\begin{abstract}
Este artículo presenta un análisis sobre los factores que inciden en el fracaso y en el riesgo de abandono escolar en la enseñanza secundaria obligatoria, como aspectos relevantes a considerar en la educación inclusiva. Se conceptualizan ambos términos y se toma en consideración el Modelo Ecológico de Bronfenbrenner (1987) para clasificar los factores que afectan al rendimiento escolar y que pueden desencadenar las problemáticas analizadas. Se hace referencia fundamentalmente a factores del centro escolar y del ambiente familiar como microsistemas de referencia del alumnado, así como a otros vinculados a la cooperación entre las familias y el centro escolar (mesosistema). Otros factores relacionados con el exosistema y el macrosistema se encuentran también referenciados. Se finaliza el artículo ofreciendo un conjunto de sugerencias de actuación relacionadas con todos los sistemas mencionados que pueden contribuir a prevenir las problemáticas analizadas y a promover la inclusión educativa del alumnado afectado por ellas.
\end{abstract}

Descriptores: Fracaso escolar, Abandono escolar, Educación familiar, Cooperación centro escolar-familia, Modelo ecológico de Bronfenbrenner.

\begin{abstract}
This paper presents an analysis of the factors affecting school failure and dropout in secondary school, as relevant matters to be considered in inclusive education. Both terms are conceptualized. The Ecological Model by Bronfenbrenner (1987) is took into account to classify factors affecting school achievement, which might relate to the both school problems analyzed. Special attention is given to school and family factors as students' microsystems, as well as to other factors related to schools and families partnerships (mesosystem). Other factors regarding the exosystem and macrosystem are also mentioned. The paper ends with some suggestions to both prevent school failure and dropout and to promote the inclusion of the students affected by them.
\end{abstract}

Keywords: School failure, Dropout, Family education, School-family partnerships, Bronfenbrenner's ecological model.

*Contacto: alvarezblucia@uniovi.es 


\section{Introducción}

En la era de la información, de la comunicación y de la sociedad globalizada se aspira a que la educación sea verdaderamente inclusiva (Alonso, 2014; Booth, Simón, Sandoval, Echeita y Muñoz, 2015ํㅣ y Escudero, 2005). Esta pretensión requiere realizar análisis, reflexiones y transformaciones sobre las prácticas educativas y los agentes que configuran la comunidad escolar y social (López, 2009) con el objetivo de lograr educación equitativa, justa y de calidad (Townsend, 2007) articulada sobre una "mayor participación en el aprendizaje, las actividades culturales y comunitarias y reduciendo, por tanto, la exclusión” (Booth et al., 2015, p. 11).

A este respecto, siguiendo lo expuesto en el Instituto Nacional de Evaluación Educativa (2013) la estrategia Europa 2020 incluye un apartado sobre los sistemas educativos, entre cuyos objetivos sobresale reducir la tasa de abandono escolar temprano, que ascendía en España en 2014 a 25.6\% entre los varones y a $18.1 \%$ en las mujeres (INE., 2015). Aun observándose una tendencia gradual a la reducción de estos porcentajes, parece difícil conseguir llegar en España al esperado 10\% en el año 2020, puesto que la cifra actual llega casi a duplicar las tasas de prácticamente todos los países de la Unión Europea.

Diversos autores han identificado factores e indicadores de riesgo que reducen la probabilidad de que alumnado alcance un rendimiento académico satisfactorio e incrementan la de que aumente el riesgo de abandono. Estos factores se encuentran asociados al: 1) ámbito personal: capacidad intelectual y rasgos de personalidad, competencias emocionales, etc.; 2) ámbito escolar: factores referentes al alumnado, a la acción docente, a la administración y gestión de centros, etc. y 3) ámbito familiar: tipología familiar -con especial referencia a las familias monoparentales-, familias inmigrantes (Santín, 2006), nivel de estudios y situación laboral de los progenitores, recursos educativos y clima de convivencia en el hogar, atención y seguimiento parental de los hijos, etc. (Calero, Choi y Waisgrais, 2010).

Fernández, Mena y Riviere (2010) y Puig (2003) sostienen que los principales factores asociados al riesgo de abandono escolar son: 1) el absentismo, 2) el bajo rendimiento académico, 3) la repetición de curso, 4) baja autoestima, 5) escaso o nulo interés hacia el estudio y 6) inadecuada adaptación social.

Por su parte, Pedró (2012) alude a la significatividad e impacto social de esta problemática, y a las medidas específicas para afrontarla que se contemplan en la Estrategia Europa 2020: 1) prevención del fracaso y abandono escolar prematuro, especialmente en el alumnado cuya lengua sea distinta a la del país en que está escolarizado y que muestre una trayectoria de repetición de curso; 2) actuación ante episodios de absentismo y bajo rendimiento académico y 3) fomento de una educación inclusiva y compensatoria fundamentadas en la igualdad de oportunidades del alumnado. En estas medidas se consideran factores no solo centrados en el alumnado, sino también en los profesionales, en los procesos y prácticas educativas, en el núcleo familiar y en el propio sistema

\footnotetext{
${ }^{1}$ Por motivos de extensión de este documento, remitimos al lector al siguiente enlace: bit.ly/1PdSj5J En el mismo se recogen algunos de los aspectos sobre los que debe articularse una educación verdaderamente inclusiva que vele por garantizar una equidad y calidad educativa. En este caso, como valiosa excusa y marco de autoanálisis sobre la idiosincrasia de cada contexto educativo y, en consecuencia de mejora del mismo, presentamos una versión actualizada y traducida al castellano del Index for Inclusion (Booth y Ainscow, 2011).
} 
educativo (Cernadas y Pérez-Marsó, 2014). A este respecto se suelen criticar las estadísticas sobre abandono y fracaso escolar que se centran solo en la medición de aprendizajes cognitivos, fácilmente cuantificables, sin tener en cuenta también otros aspectos personales, emocionales y biográficos del alumnado (Escudero, González y Martínez, 2009).

Por ello, en este artículo se analiza esta problemática socioeducativa (Gimeno, 2005) desde un enfoque multicausal y multidimensional tomando en consideración tanto factores que afectan al propio estudiante como a los sistemas ambientales en que éste interactúa (Navarro, 2001; Zelmanovich y Levinsky, 2012).

\section{Aproximación conceptual al concepto de fracaso y de abandono escolar}

En la literatura científica se advierte el empleo de una pluralidad de términos para aludir al abandono del sistema educativo por parte de los estudiantes. A modo de ejemplo, algunas expresiones utilizadas en España son "abandono temprano" (INE, 2015) o "abandono educativo temprano" (Ministerio de Educación, Cultura y Deporte., 2014), que se suelen relacionar con el fracaso escolar.

Investigadores como Fernández y colaboradores (2010) y Herrero (2001) subrayan que tanto el fracaso como el abandono escolar son fruto de una progresiva desvinculación de los estudiantes de las instituciones escolares, lo que se entiende como un obstáculo para la inserción e inclusión social y laboral de este alumnado.

El fracaso escolar es definido por Aristimuño (2015, p. 110) como la "no aprobación en el tiempo previsto -ya sea por rezago, repetición o desafiliación- en el tramo educativo que se está transitando". Marchesi (2003) comparte esta idea al entender que los estudiantes etiquetados de "fracaso escolar" son quienes "al finalizar su permanencia en la escuela no han alcanzado los conocimientos y habilidades (...) necesarios para manejarse de forma satisfactoria en la vida social y laboral o proseguir sus estudios" (p. 8). Conforme esta definición, la sociedad se enfrenta a un fenómeno preocupante que incita a pensar en la necesidad de realizar diagnósticos y seguimientos preventivos y periódicos del alumnado cuando todavía no presenta problemas académicos relevantes y no solamente cuando ya se han detectado claros indicios de fracaso como la repetición de curso. Parece conveniente actuar en este sentido desde el inicio de la escolarización obligatoria, y especialmente en el tránsito de una etapa educativa a otra: Educación Infantil-Educación Primaria y Educación Primaria-Educación Secundaria Obligatoria.

Esta línea de actuación preventiva parece conveniente, dadas las dos variantes de fracaso escolar señaladas por Carbonell y Peña (2006): a) fracaso escolar Primario, advertido en los primeros cursos de escolarización y que frecuentemente se asocia con dificultades de aprendizaje de diversa naturaleza y gravedad, y b) fracaso escolar Secundario, acaecido en momentos evolutivos especialmente críticos como la adolescencia, o coincidentes con circunstancias estresantes o disfuncionales en el ámbito familiar o escolar.

En este documento se empleará el término “abandono escolar prematuro” para designar al alumnado que en España cesa voluntariamente sus estudios por diversas razones (desmotivación, absentismo, dificultades de aprendizaje, repetición de curso, etc.) al 
alcanzar la edad ${ }^{2}$ mínima legal de 16 años sin haber obtenido la titulación de Graduado en Educación Secundaria Obligatoria. Previamente a que se produzca el abandono, se hablará de "riesgo de abandono escolar".

\section{Fracaso y abandono escolar desde el modelo ecológico de Bronfenbrenner}

En este epígrafe se analizan algunos factores significativos asociados al fracaso y al riesgo de abandono escolar. Como ya comentamos, ambos fenómenos están estrechamente vinculados, puesto que la reiterada obtención de bajos rendimientos, desmotivación ante el estudio, baja implicación en las actividades del aula, etc. pueden conducir al alumnado a cesar prematuramente en sus estudios (Mora, 2010).

Entendemos que identificando los factores que pueden incidir negativamente sobre el progreso escolar del alumnado desde distintos ámbitos de interacción (familia, centro educativo, sociedad, etc.) se podrán promover en los centros actuaciones que permitan prevenir el abandono académico (Álvarez, 2006 y Martínez y Álvarez, 2005) y convertirse así en centros más eficaces (Murillo, 2005).

Existe un amplio volumen de autores que han tratado de identificar las causas de esta problemática (Álvarez, 2012 y 2006; Asensio, 1994; Balzano, 2002; Bermejo, Martín y Ayala, 2006; Del Burgo, 2002; Fernández, 2005; Fernández et al., 2010; Fullana, 1998; Grau, Pina y Sancho 2011; Lozano, 2003; Marchesi, 2012 y 2009; Marchesi, Martínez y Martín, 2004; Navarrete, 2007; Portillo-Torres, 2015; Ramo, 2000; Sánchez, 2001; Soler, 2003; Taborda, Formosinho y Fonseca, 2007, entre otros). Si bien existen algunas discrepancias entre estos autores sobre la priorización de algunos factores causales, es más evidente el consenso entre ellos en lo que respecta a la relación de aspectos a considerar: "contexto económico y social, dedicación y expectativas de las familias, gasto público en educación, tiempo de enseñanza, organización y funcionamiento de los centros docentes, estilo de enseñanza (...) e interés de los alumnos" (Marchesi, 2003, p. 5). Estos aspectos pueden analizarse desde el modelo Ecológico del Desarrollo Humano de Bronfenbrenner (1987), también conocido como Sistema de Sistemas sistema ontogenético, microsistemas, exosistema, macrosistema y mesosistema. El concepto de sistema remite a interrelación, interdependencia e influencias recíprocas entre diferentes elementos de un conjunto o espacio convivencial. De manera resumida, Musitu, Estévez y Jiménez (2010) han expuesto los supuestos sobre los que se articula un sistema social:

- El funcionamiento sistémico se concibe como el resultado holístico de interacciones multiplicativas, bidireccionales y recíprocas entre las diferentes esferas que entran en relación y no como la mera suma de las interacciones acaecidas dentro de un sistema (López, Utrilla y Valiño, 2006).

- Reticencia al cambio (homeostasis). Cualquier modificación en el interior de un sistema suele generar rechazo y resistencia por parte de sus miembros,

\footnotetext{
2 A este respecto autores como Fernández et al. (2010) incluyen un interesante matiz referido a la edad del estudiante, sosteniendo que antes de cumplir los 16 años se trata realmente de un abandono del alumno por parte de la institución y no de una desvinculación escolar del propio estudiante. Entre aquellos que tienen 16 y 17 años sucede que el abandono se presenta como una opción de quien ostenta la tutela o Patria Potestad. Así las cosas, el abandono voluntario se entendería como libre elección solo entre el alumnado mayor de 18 años.
} 
siendo imprescindible que quien asuma la iniciativa del cambio desarrolle estrategias y habilidades que le permitan afrontar y defenderse de dichas actitudes y comportamientos de resistencia.

- Puesto que el sistema se rige por interacciones sociales, éstas representan la distribución y asunción de distintos roles que posibilitarán el correcto funcionamiento del conjunto.

- Generación de distintas expectativas y comportamientos que condicionarán el ejercicio de los respectivos roles conferidos y asumidos por los miembros del sistema.

Choque (2009) ha tomado como referencia el Modelo Ecológico de Bronfenbrener para extenderlo al ámbito educativo, refiriéndose a él como ecosistema educativo, entendido como "el conjunto de personas y organizaciones constituyentes del microsistema, el mesosistema, el exosistema y el macrosistema, que de manera interrelacionada interactúan con la finalidad de lograr los objetivos educativos en un determinado contexto social" (Choque, 2009, p. 3).

Además de los sistemas mencionados, se añade el sistema ontogenético, es decir, el individuo en desarrollo, que en el ámbito educativo se corresponde con los estudiantes.

En los siguientes epígrafes se ofrece un análisis de la influencia que los distintos sistemas en que el alumnado interactúa ejercen en su proceso de adaptación al ámbito educativo, pudiendo derivar en situaciones de fracaso y abandono escolar.

\section{Los microsistemas familia y centro escolar y su interdependencia (mesosistema)}

A grandes rasgos puede definirse el microsistema como los entornos "más próximos a los menores en proceso de desarrollo" (Martínez y Pérez, 2004, 93). De acuerdo con Choque (2009, p. 3) son un "escenario concreto que comprende un entorno físico, emocional y de interrelación que se da entre las personas”. En este sentido, la familia y el centro escolar son microsistemas de donde el individuo recibe las influencias más poderosas (valores, creencias, actitudes, roles, normas, etc.) y con los que mantiene sus primeras relaciones recíprocas y directas. Trasladando el concepto de microsistema al análisis del fracaso y abandono escolar parece relevante analizar los factores de influencia que proceden de la familia y del centro escolar.

\subsection{El centro escolar como factor de influencia en el fracaso y abandono escolar}

En el centro educativo se aplican las disposiciones y ordenamiento general del sistema educativo; por ello, parece oportuno reflexionar sobre alguno de los aspectos que lo configuran y de cuya calidad de funcionamiento puede resultar que se facilite o dificulte el proceso de enseñanza-aprendizaje, pudiendo emanar de este último caso episodios de fracaso, absentismo y abandono escolar que incitan a un debate abierto a la esfera social y de la comunidad escolar en aras de conocer las principales repercusiones que se derivan de los mismos (UNESCO, 2012). Los informes internacionales sobre resultados de la educación como los de PISA refuerzan la idea de que no existe un único aspecto explicativo del rendimiento escolar y de las diferencias que se encuentran entre los países. 
De modo que el fracaso y el abandono escolar no han de ser entendidos como derivados solo de factores asociados a los estudiantes, aunque ésta sea la perspectiva más frecuente que contribuye a "dar a las escuelas una excusa ante su falta de éxito con el que abandona" (González, 2006, p. 6). En este sentido, cabría pensar que la concurrencia de prácticas organizativas y docentes inadecuadas podría influir en el surgimiento del fracaso escolar, que, de no ser afrontado adecuadamente, podría derivar en riesgo de abandono escolar. González (2001, p. 51) apunta que "las normas que regulan la actividad educativa, las formas concretas de organización de esa actividad y la práctica diaria puede ser (...) causa directa de fracaso y (...) de otros fenómenos asociados como el absentismo, frustración y conductas más o menos negativas”. De ahí la conveniencia de evaluar la organización y los procesos educativos y de convivencia que tienen lugar en las instituciones académicas.

Entre estas actuaciones, Marchesi y Pérez (2003, p. 41) citan: "el liderazgo del equipo directivo, el ambiente favorable de aprendizaje, la existencia de un proyecto compartido, la organización eficiente de la enseñanza en el aula, la participación de los padres y de los alumnos, el seguimiento del progreso de los alumnos y la evaluación de la escuela”. Por su parte, Vaquero (2005, p. 1460) apunta: "el nivel socioeducativo de los estudiantes, la facilidad de relación entre profesores y alumnos, la disponibilidad de recursos educativos en la escuela y la familia y el clima escolar”.

El Community Health Systems Resource Group (2005, p. 14) añade también: “un sistema de disciplina ineficaz; ausencia de un adecuado proceso de consejo/asesoramiento, clima escolar convivencial, inexistencia de curriculum relevante, estrategias instruccionales pasivas, falta de consideración hacia los estudios de aprendizaje de los estudiantes, detenciones y/o suspensiones, falta de evaluación y apoyo al alumnado con discapacidad”.

En definitiva, prevenir los problemas de fracaso y abandono escolar para alcanzar eficacia y éxito en las escuelas (Townsend, 2007; Wikeley, Stoll y Lodge, 2002) no solo dependerá de cuestiones relativas al alumnado, sino también de otras de índole material, docente innovaciones docentes, metodología docente, oferta de oportunidades de aprendizaje...(UNICEF, 2012), convivencial y del esfuerzo compartido por toda la comunidad educativa. Este último aspecto es relevante; por ello, es conveniente que el centro se abra a la comunidad para aunar esfuerzos y recursos con las familias y con otras entidades sociales del entorno inmediato (Bolívar, 2008).

En el entorno comunitario ("exosistema” en el modelo ecológico de Bronfenbrenner) pueden encontrarse opciones de formación y aprendizaje complementario al que hace el alumnado en los centros escolares; esto puede ser de interés para aquellos que se encuentran desmotivados en la institución educativa y que, por ello, pueden llegar al fracaso o al abandono escolar (Tarabini y Curran, 2015).

El centro educativo, al igual que la familia, el barrio, el grupo de iguales o los medios de comunicación, es un importante agente de socialización. Como indica González (2006, p. 7), aunque "las escuelas pueden hacer poco en lo que respecta a las circunstancias socioeconómicas y características de los alumnos (...), sí tienen la obligación de proporcionar ambientes educativos efectivos y ricos para todos los alumnos”. Además de la faceta docente curricular, el centro escolar transmite al alumnado valores, creencias, modos de interacción y conducta, en ocasiones muy diferentes a los forjados en la familia. Por este motivo compartimos lo expuesto por Del Burgo (2002, p. 81) sucediendo que: "la escuela también puede y debe brindar ayuda y orientación a los padres más allá de la estricta información académica, dado que la familia, como otras instituciones sociales, se 
ve sometida a cambios tan rápidos y complejos que a veces son difíciles de afrontar y asimilar".

Numerosas investigaciones (Epstein, 2011; Symeou, Martínez-González y Álvarez, 2012) han demostrado que ello contribuye a prevenir el fracaso y el riesgo de abandono escolar. En último término, se trata de garantizar que: "las acciones (ejercidas desde el centro educativo) se encaminarán a preparar al alumnado y a su familia para que asimilen la nueva cultura escolar y puedan formar parte de ella" (Valls, 2003, p. 65).

\subsection{La familia como factor de influencia en el fracaso y abandono escolar}

El primer escenario de socialización de los niños y jóvenes es la familia, institución que contribuye a su desarrollo óptimo, a su educación y aprendizaje (Ceballos, 2006). Son muchos los aspectos a considerar en el estudio de la familia como agente educativo y de socialización que pueden resultar relevantes en el análisis del fracaso y abandono escolar (Suárez, Tuero-Herrero, Bernardo, Fernández, Cerezo, González-Pienda, Rosario y Núñez, 2011). De entre ellos, el Community Health Systems Resource Group (2005, p. 14) hace referencia a los siguientes: a) En referencia al microsistema familiar: "bajo nivel sociocultural, pertenencia a grupos minoritarios, el propio hecho de ser mujer, estrés existente en el hogar, dinámicas de convivencia familiar y asunción de roles por parte de los adultos"; b) en referencia al mesosistema: "inexistencia o escasez de apoyo social para permanecer vinculando con la institución escolar, conflicto y falta de cooperación entre la familia y el centro escolar" y c) en referencia al exosistema: "ciertas características comunitarias".

De acuerdo con las indicaciones señaladas por este grupo, tanto los comportamientos parentales de implicación en la educación de los hijos en el hogar, como los relativos a la cooperación entre familia y centro escolar, constituyen medidas preventivas y paliativas del fracaso escolar y del abandono temprano de los estudios.

Las experiencias de colaboración entre los centros escolares y las familias son altamente positivas para prevenir estas problemáticas. Las investigaciones realizadas al respecto constatan que facilitan la integración e inclusión educativas (Escudero et al., 2009) y beneficia también al alumnado que presenta ya éxito escolar. Sobre esto, Musitu y Cava $(2001,133)$ indican que la participación activa de los padres y madres en el centro escolar "incide de forma positiva en el rendimiento académico, las habilidades sociales, la autoestima, el menor absentismo, los hábitos de estudio y las actitudes positivas hacia la escuela de los hijos". Es altamente significativo el papel que los padres y madres juegan en el desarrollo de la autoestima de los hijos, de sus habilidades sociales y de la actitud y motivación hacia el estudio (Moreno, 2010; Muñoz, 2009). Precisamente, las competencias de autoestima y autoconcepto son dos de las dimensiones personales que más frecuentemente se ven afectadas y mermadas por el fracaso y abandono escolar en la etapa adolescente (Álvarez, 2012).

En los siguientes apartados se analizan algunos indicadores que relacionan el fracaso escolar con la familia, que ofrecen pautas para el análisis y la reflexión. En palabras de Santín (2006, p. 199), "la producción de educación en un niño es un proceso muy complejo en el que distintas variables y agentes interrelacionan, pero donde la familia es la verdadera piedra angular para evitar el fracaso escolar". 


\subsubsection{Nivel de estudios de los padres y madres}

Las investigaciones que analizan las relaciones entre el nivel de estudio de los padres y madres y el rendimiento escolar suelen concluir que "a mayor nivel educativo de los padres, menor es el nivel de fracaso de los hijos” (Fernández et al., 2010, p. 75). De acuerdo con Murillo (2007, p. 120) el nivel de estudios materno es "el indicador de nivel cultural con mayor poder explicativo del rendimiento de los alumnos", presentándose de este modo como un elemento facilitador del progreso educativo de los hijos al apreciarse que los hijos avanzan mejor en su proceso de aprendizaje cuanto más elevado es el nivel formativo de aquel. Asimismo, en referencia a la clase social, se constata una correlación positiva con el abandono escolar, que lleva a Taborda y colaboradores (2007, p. 182) a "invocar razones culturales para justificar, tanto el menor índice de suceso (éxito) escolar como la escolaridad más reducida de los alumnos de medios desfavorecidos".

Esta consideración es compartida por Pérez-Díaz, Rodríguez y Sánchez (2001) en su investigación sobre la implicación educativa familiar, subrayándose que cuanto mayor es el nivel educativo de los padres y madres, menor es la cantidad de asignaturas que el hijo suspende: el $76 \%$ de los hijos cuyos progenitores poseen estudios universitarios aprueban todas las asignaturas frente a tan sólo el 36\% de alumnos cuyas familias tienen estudios primarios incompletos y el $41 \%$ de quienes han completado la educación Primaria. En contraposición, el mayor número de asignaturas suspensas (4 o más) se localiza en alumnado con familias con estudios primarios completos (26\%) e incompletos (25\%), frente a tan sólo un 4\% de alumnado cuyos padres y madres tienen estudios universitarios.

Corroborando estos datos, Meil (2006, p. 99) también ha apreciado que "el nivel educativo de los padres juega un papel importante (...) en función de si tienen estudios primarios o menos, o tienen estudios más allá de los primarios". Por su parte, Torío, Hernández y Peña (2007) matizan que cuanto mayor es el nivel de estudios materno, y mayor es su nivel cultural y social, más elevado es el porcentaje de hijos estudiantes de la educación secundaria obligatoria (ESO) que indica que seguirán estudiando tras concluir esta etapa. Además, cuanto menor es el nivel de estudios paterno, mayor es el número de los adolescentes que piensan trabajar en el futuro próximo en vez de estudiar.

El nivel educativo de los padres y madres es importante para entender, por ejemplo, sus distintos grados de habilidades para ayudar a sus hijos con sus tareas escolares como, por ejemplo, hacer consultas bibliográficas o informáticas, manejar programas básicos de ordenador, etc. De igual modo, el nivel de estudio parental condiciona el código lingüístico que utilicen (elaborado o restringido), el valor que confieren a la educación, el desarrollo de expectativas académicas, la consideración que hacen de la labor docente, etc., que influye en las expectativas que generan sus hijos y en la imitación de sus comportamientos.

Por otro lado, vinculando el nivel de estudios parental con la repetición de curso, PérezDíaz y colaboradores (2001) señalan que el porcentaje de alumnos que repite curso es inversamente proporcional al nivel de estudios de sus familias, de modo que a mayor nivel, menor porcentaje de repetición. Así, en la muestra seleccionada por estos autores $(\mathrm{N}=2519)$, únicamente repiten curso el $5 \%$ de los hijos cuyos padres y madres poseen estudios universitarios, porcentaje que se eleva hasta el $16 \%$ en familias con estudios primarios incompletos. No obstante, a pesar de esta influencia que tiene el nivel formativo y cultural de los padres y madres en el éxito escolar, es preciso resaltar también la importancia de las actitudes parentales y de su expectativas positivas hacia los hijos para 
facilitar su correcta adaptación a los estudios y al centro escolar (Asensio, 1994). Estos dos factores no siempre están asociados al nivel de estudios de los progenitores.

\subsubsection{Expectativas parentales hacia los hijos y hacia su educación}

En estrecha relación con la clase social y nivel de estudios de los padres y madres, cabe analizar las expectativas que éstos desarrollan hacia la educación y el éxito escolar de sus hijos (UNICEF, 2012). Habitualmente, los padres y madres sienten desconcierto, duda e incluso culpabilidad si sus hijos presentan un reiterado fracaso escolar cuando son adolescentes y se encuentran cursando la etapa de la educación secundaria obligatoria porque esto constituye un riesgo de abandono de los estudios (Pérez de Pablos, 2003). Los resultados académicos de los hijos son fuente habitual de preocupación familiar (Santín, 2006), al entender "la obtención de los correspondientes títulos como mecanismo para lograr el éxito y promoción social” (Meil, 2006, p. 92).

De acuerdo con la literatura científica consultada, no existe duda sobre la influencia que ejercen las expectativas parentales en el rendimiento académico de los hijos (Marchesi y Martín, 2002), así como el valor que los padres y madres conceden a la educación y al estudio (Castejón y Pérez, 1998) y la atención y ayuda que prestan a sus hijos a este respecto (Santín, 2006).

A esta conclusión llegan Marina (2010) y Marchesi y Pérez (2003,37) en una investigación en la que constatan que: "las expectativas de los padres manifiestan una significativa influencia en los resultados de los alumnos prácticamente en todas las áreas curriculares y en todos los cursos de la ESO”. Por su parte, Rosario, Mourão, Núñez, González-Pienda y Solano (2006, 171-172) concluyen en uno de sus estudios que estas expectativas afectan indirectamente al rendimiento del estudiante "a través de su incidencia sobre variables personales tales como el autoconcepto (...), el patrón típico de atribución de la causalidad sobre éxitos y fracasos académicos (...), etc.”. Por tanto, las expectativas que los padres y madres tienen hacia sus hijos pueden repercutir en su desempeño curricular, en sus inclinaciones profesionales y en sus logros académicos a través de una proyección consciente o inconsciente de éstas en la percepción que desarrollan los propios hijos hacia su futuro (Torío et al., 2007).

Dada la relevancia de los aspectos comentados vinculados al ámbito familiar, se hace necesario promover una educación inclusiva y compensatoria en la que, independientemente de la situación socioeconómica y cultural familiar (Fernández, 2009), el alumnado disfrute de una auténtica igualdad de oportunidades para tener éxito en los estudios y permanecer en el sistema escolar (Olmos y Mar, 2013), contribuyéndose de esta manera a una mejora en el capital humano (estrechamente relacionado con el rendimiento académico), social y cultural (Symeou, 2006) como vehículos del desarrollo sostenible de un país construido sobre pilares como la cohesión social, prosperidad económica e inclusión (Escudero, 2005).

\subsubsection{La implicación educativa familiar}

Definir y operativizar el concepto de implicación parental no resulta fácil por la multiplicidad de aspectos que engloba. Fan y Chen (2001, p. 3) entienden que este constructo aglutina los siguientes comportamientos, actitudes y prácticas parentales: "expectativas parentales sobre el aprovechamiento académico de sus hijos/as, comunicación parental con los hijos, participación parental en las actividades escolares, 
comunicación parental con el profesorado sobre los hijos y reglas parentales existentes en el hogar y referidas a la educación”.

Fomentar una frecuente y efectiva implicación de los padres y madres en la educación y aprendizaje de sus hijos (González-Pienda y Núñez, 2005) con el apoyo de la institución educativa es fundamental, sobre todo cuando se presentan disfunciones en la convivencia familiar (Navarrete, 2007) y cuando el alumnado manifiesta problemas de fracaso escolar. Investigadores como Deslandes y Bertrand (2005) y Pérez, Rodríguez y Fernández (2009) han analizado la implicación familiar y su relación con el rendimiento escolar considerando dos vertientes de la misma: la implicación parental en el hogar (involvement at home) y en el centro escolar (involvement at school). A este respecto, cabe comentar algunas actuaciones parentales que resultan relevantes:

\section{a) Ayuda parental en la realización de las actividades de aprendizaje en el hogar}

Los docentes suelen establecer deberes para realizar en el hogar con el objetivo de que el alumnado refuerce fuera del horario lectivo el aprendizaje iniciado en clase. De acuerdo con Meil (2006, p. 98) "la ayuda con los deberes se convierte en un indicador adicional de los padres en la educación de sus hijos (...), su análisis permite profundizar en el papel actual de los padres en la educación de sus hijos, en la importancia que le dan y en el grado de implicación que tienen”.

Pérez-Díaz et al. (2009) han encontrado que es elevado el porcentaje de progenitores que dialogan con sus hijos sobre su proceso educativo ( $85 \%$ dice hacerlo varias veces a la semana), así como el número de aquellos que prestan su ayuda en los trabajos escolares de sus hijos ( $56 \%$ lo hace siempre o casi siempre). Asimismo, estos autores constatan la existencia de una asociación directa entre el número de suspensos y la frecuencia parental de ayuda en los deberes, sucediendo que a mayor implicación, menor número de suspensos.

\section{b) Acompañamiento, atención y tiempo para estar juntos}

Con independencia del nivel educativo que tengan los padres y madres, resulta satisfactorio y positivo para los hijos que aquellos valoren su esfuerzo, les animen y les muestren su apoyo y confianza en sus capacidades y potencialidades para aprender y progresar en su proceso de aprendizaje. También es importante que los padres y madres fomenten en sus hijos hábitos de estudio (Pérez de Pablos, 2003) como la regularidad, organización y distribución de tiempos de ocio y de formación, habilidades de trabajo intelectual, fomento de la lectura, etc. Estas prácticas parentales no tienen por qué realizarse solo cuando se hacen los deberes escolares, sino también en la cotidianidad de la convivencia familiar, transmitiendo a los hijos una expectativa de apoyo y cercanía. Estas conductas parentales están al alcance de todas las familias, con independencia del nivel de estudios de los padres y madres (Redding, 2006).

\section{c) Participación de los padres y madres en el centro escolar}

Esta participación se contempla en la legislación y políticas educativas. En muchos casos, se organiza a través de los Consejos Escolares y de las Asociaciones de Padres y Madres del Alumnado de los centros escolares. Ambas entidades suelen proponer a las familias participar en sus órganos de representación colectiva o en actividades extraescolares, celebraciones deportivas y culturales, así como en acciones formativas específicas para las familias. Esta participación es relevante porque permite un contacto cercano y frecuente entre las familias y los centros, y transmite a los hijos y al colectivo docente la idea de que 
los padres y madres procuran estar informados de lo que acontece en el centro y que se preocupan por la educación de sus hijos.

No obstante, Marchesi (2000) advierte sobre la reducida participación parental en los centros, especialmente en la etapa de la educación secundaria, aunque los padres y madres sí asistan en ocasiones a charlas informativas o a reuniones con el profesorado. Las dinámicas cotidianas actuales en el ámbito familiar y laboral dificultan en muchas ocasiones que los padres y madres dispongan del tiempo necesario para participar en la vida escolar; esto les resta oportunidades para fomentar relaciones más directas con el profesorado, con otras familias, para conocer más de cerca las exigencias actuales del sistema escolar y las dificultades que pueden experimentar sus hijos en su proceso de aprendizaje académico. Esta falta de tiempo para conectar con el centro puede llevar en ocasiones a los padres y madres a delegar en el profesorado ciertas responsabilidades educativas que les corresponden a ellos (Marchesi, 2000; Roldán, 2008) aun cuando son conscientes de que "no se puede dejar en manos de otras instituciones aquello que es responsabilidad personal y social de los padres” (López y Martín, 2008, p. 47).

Los resultados obtenidos por Robledo y García (2006, p. 229) en investigaciones llevadas a cabo sobre el grado de satisfacción de los padres y madres cuando participan en los centros escolares indican que, independientemente de la modalidad de participación, la satisfacción familiar es notable: "la mayoría de los padres cuyos hijos presentan dificultades, bajo rendimiento o fracaso escolar, están satisfechos con los servicios educativos (...) así como con los servicios profesionales”. Por su parte, García y Delgado (1994, p. 116), en una investigación realizada sobre el desempeño profesional docente han identificado algunos aspectos que solicitan las familias al centro escolar y al profesorado: "eliminación de contenidos innecesarios de programas (27\%), mejora y perfeccionamiento de los métodos y técnicas de enseñar (20\%) y el que los profesores (...) se adecuen al ritmo de cada alumno (19.3\%)".

Para finalizar, cabe referenciar algunos efectos positivos que, de acuerdo con García (1996), se derivan de la participación educativa familiar: a) relación directa entre la implicación familiar y el éxito académico; b) relación directa entre la implicación familiar, la asistencia de los hijos al centro y el desarrollo de actitudes positivas hacia éste. También se ven favorecidos el autoconcepto y el comportamiento; c) la implicación parental afecta positivamente a los propios padres, a los profesores, a la comunidad, etc., siendo más satisfactoria cuanto más comprensiva y prolongada sea en el tiempo; d) la implicación será más exitosa en la medida en que coincida con los años escolares iniciales del alumno. Esta afirmación es especialmente relevante cuando se sabe que "las mayores diferencias en la existencia de ayuda depende (...) de la edad de los hijos, de forma que a mayor edad, menor ayuda, aunque también (...) mayor número de suspensos" (Meil, 2006, p. 100).

El análisis realizado indica la conveniencia de reforzar la implicación familiar en los estudios de los hijos por su influencia en el proceso de adaptación de éstos al centro escolar, así como en su proceso de aprendizaje y en otras facetas de su desarrollo personal, afectivo, relacional y de adquisición de valores y competencias. 
Tabla 1. Sugerencia de actuación para prevenir y afrontar el fracaso y el riesgo de abandono escolar

\begin{tabular}{|c|c|c|}
\hline 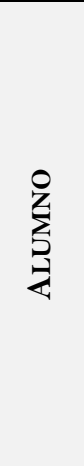 & 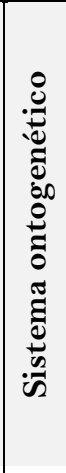 & $\begin{array}{l}\text { Desarrollar una atención educativa personalizada con el alumnado atendiendo a los } \\
\text { diferentes perfiles y necesidades que se detecten. } \\
\text { Promover el desarrollo integral del alumnado considerando que las cuestiones } \\
\text { emocionales adquieran la misma relevancia que otras de naturaleza curricular. } \\
\text { Convendría potenciar especialmente la conciencia de que son capaces de progresar } \\
\text { personal y académicamente favoreciendo con ello su motivación e implicación en los } \\
\text { estudios y en el centro. } \\
\text { Controlar las ausencias del alumnado al centro escolar contando con la colaboración } \\
\text { de profesionales de servicios a la comunidad, policías tutores, etc., informando a las } \\
\text { familias. } \\
\text { Fomentar el autoconcepto y autoestima del alumnado, al verse altamente afectados } \\
\text { por bajas calificaciones y repetición de curso. }\end{array}$ \\
\hline 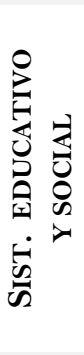 & 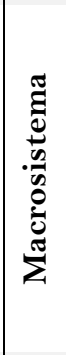 & $\begin{array}{l}\text { Promoción de políticas familiares que apoyen cultural y económicamente a las } \\
\text { familias del alumnado que así lo precise. } \\
\text { Aumentar la dotación de recursos que se destinan a los centros escolares y organizar } \\
\text { adecuadamente los que existan. } \\
\text { Cuidar la ratio docente-alumnado en cada aula incluyendo profesionales de apoyo al } \\
\text { alumnado y, si es el caso, a los padres y madres. } \\
\text { Insistir en el valor del esfuerzo, igualdad de oportunidades y promoción de pautas } \\
\text { específicas que ayuden al alumnado a afrontar de manera no traumática la transición } \\
\text { de la Educación Primaria a la Secundaria. }\end{array}$ \\
\hline 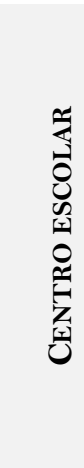 & 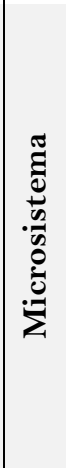 & $\begin{array}{l}\text { Promover la detección temprana de indicadores de fracaso escolar: reforzar las clases } \\
\text { de apoyo escolar en el propio centro o con las familias en aquellos casos en que se } \\
\text { detecten dificultades de aprendizaje. } \\
\text { Incidir en el aula en el desarrollo de actitudes positivas, habilidades y capacidades del } \\
\text { alumnado evitando centrarse solo en la transmisión de conocimiento y en la } \\
\text { evaluación de contenidos conceptuales. } \\
\text { Reducir la ratio en las aulas, especialmente en aquellos casos en que el alumnado } \\
\text { manifieste conflictos o problemas personales y/o familiares, dificultades de } \\
\text { aprendizaje, baja motivación e interés por el estudio. } \\
\text { Fomentar el desarrollo de programas de formación (de tipo formal e informal) que } \\
\text { promuevan la implicación y trabajo conjunto de la familia, alumnado, profesorado y } \\
\text { otras instituciones sociales. }\end{array}$ \\
\hline 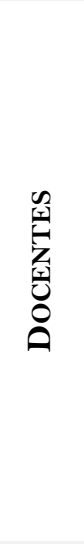 & 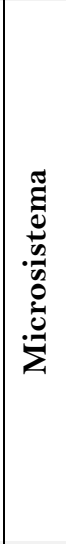 & $\begin{array}{l}\text { Fomentar la formación permanente del profesorado en metodologías docentes, } \\
\text { cuestiones psicopedagógicas, evolutivas...y especialmente, con respecto al trabajo } \\
\text { específico con alumnado en potencial riesgo de abandono escolar y con las familias } \\
\text { Proponer formas alternativas de evaluación de los estudiantes, dando importancia al } \\
\text { progreso diario en la consecución de metas de aprendizaje, valorar su implicación, } \\
\text { actitud, esfuerzo y compromiso por aprender. } \\
\text { Mejorar las metodologías de enseñanza atendiendo a la: } \\
\text { Combinación de clases magistrales con otras basadas en dinámicas de grupos y en } \\
\text { estrategias que faciliten el aprendizaje significativo, cooperativo y la motivación y } \\
\text { dinamismo del alumnado. } \\
\text { Presentación de los contenidos de enseñanza de una forma atractiva, motivadora y } \\
\text { adaptada a las necesidades e intereses reales del alumnado, contando con las } \\
\text { potencialidades de las tecnologías de la información y la comunicación } \\
\text { Reconocer la función social que realiza el profesorado }\end{array}$ \\
\hline 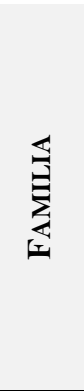 & 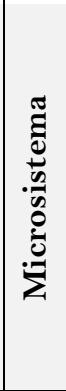 & $\begin{array}{l}\text { Fomentar contactos frecuentes con el centro escolar al que asisten los hijos para estar } \\
\text { informados de su progreso académico, actividades de aprendizaje, posibles problemas, } \\
\text { etc. } \\
\text { Aconsejar y estimular a las familias sobre la necesidad de apoyar y supervisar a sus } \\
\text { hijos en los estudios } \\
\text { Coordinar el centro educativo con los Servicios Sociales para atender necesidades de } \\
\text { las familias o déficits educativos, económicos y/o laborales que éstas puedan } \\
\text { manifestar } \\
\text { Ofrecer a las familias formación e información sobre competencias parentales para } \\
\text { lograr una acción educativa más eficaz con sus hijos, en especial con los adolescentes. }\end{array}$ \\
\hline
\end{tabular}

Fuente: Elaboración propia. 
Además de la importancia que adquiere la familia, es preciso considerar la relevancia de otros entornos en los que se desenvuelve el estudiante dentro del exosistema del Modelo Ecológico, donde se producen procesos importantes para su formación a través de la educación no formal: municipio, servicios de salud, clubes, asociaciones, etc. (Choque, 2009). Sin embargo, como expone este autor, tradicionalmente se ha explicado el éxito y fracaso escolar mediante referencias al microsistema de la familia y del centro escolar y a sus interacciones (mesosistema) por ser entornos más cercanos e inmediatos al alumnado.

Una vez revisados algunos de los factores ambientales relevantes que inciden en el fracaso y abandono escolar, finalizamos presentando algunas sugerencias de actuación para prevenir estos problemas (tabla 1), organizándolas en los sistemas del modelo ecológico que se ha tomado como referencia en este trabajo (Álvarez, 2012, 2006; Martínez y Álvarez, 2005; Portillo-Torres, 2015).

\section{Referencias}

Alonso, J. M. (2014). Política social europea. Madrid: UNED.

Álvarez, L. (2006). Familia y abandono escolar. Importancia de la implicación familiar en el proceso educativo. Madrid: Ediciones Cinca.

Álvarez, L. (2012). Análisis del riesgo de abandono escolar desde la perspectiva del alumnado, del profesorado y de las familias. Identificación de necesidades educativas (Tesis Doctoral). Universidad de Oviedo, Oviedo.

Aristimuño, A. (2015). El fracaso escolar, ¿fracaso de quién? La modificación del concepto de fracaso. Revista Latinoamericana Educación Inclusiva, 9(1), 111-126.

Asensio, J. M. (1994). Hijos con fracaso escolar. Barcelona: Grupo Editorial Ceac.

Balzano, S. (2002). Las construcciones culturales sobre el éxito y el fracaso escolar y sus implicaciones sobre los modelos educativos en la Argentina. Cultura y Educación, 3(14), 283296. doi 10.1174/11356400260366106

Bermejo, V., Martín, P. y Ayala, C. (2006). Bases neuropsicológicas del fracaso escolar. Madrid: Fugaz Ediciones.

Bolívar, A. (2008). Educación para la ciudadanía: algo más que una asignatura. Barcelona: Graó.

Booth, T., Simón, C., Sandoval, M., Echeita, G. y Muñoz, Y. (2015). Guía para la educación inclusiva. Promoviendo el aprendizaje y la participación en los centros escolares. Nueva edición revisada y ampliada. REICE. Revista Iberoamericana sobre Calidad, Eficacia y Cambio en Educación, 13(3), 5-19.

Bronfenbrenner, U. (1987). La ecología del desarrollo humano. Barcelona: Paidós.

Calero, J., Choi, A. y Waisgrais, S. (2010). Determinantes del riesgo de fracaso escolar en España: Una aproximación a través de un análisis logístico multinivel aplicado a PISA-2006. Revista de Educación, Número extraordinario, 225-256.

Carbonell, J. L. y Peña, A. I. (2006). ¿Qué hacer cuando los hijos dejan de estudiar? Educación y Futuro, 14, 97-108.

Castejón, J. L. y Pérez, A. M. (1998). Un modelo causal-explicativo sobre la influencia de las variables psicosociales en el rendimiento académico. Revista Bordón, 50(2), 171-185. 
Cooperación entre las Familias y los Centros Escolares como Medida Preventiva...

L. Álvarez y R. A. Martínez González

Ceballos, E. (2006). Dimensiones de análisis del diagnóstico en educación: el diagnóstico del contexto familiar. Revista Electrónica de Investigación y Evaluación Educativa, 12(1), 1-24. doi:10.7203/relieve.12.1.4244

Cernadas, A. y Pérez-Marsó, M. (2014). Un análisis del fracaso escolar en dos centros de Educación Secundaria. Revista de Estudios e Investigación en Psicología y Educación, 1(2), 122-131. doi:10.17979/reipe.2014.1.1.8

Choque, R. (2009). Ecosistema educativo y fracaso escolar. Revista Iberoamericana de Educación, $4(49), 1-9$.

Community Health Systems Resource Group at the Hospital for Sick Children. (2005). Early school leavers: understanding the lived reality of student disengagement from secondary school. Recuperado de http://bit.ly/zBxmNq

Del Burgo, M. (2002). El fracaso escolar. Madrid: Acento Editorial.

Deslandes, R. y Bertrand, R. (2005). Motivation of parent involvement in secondary-level schooling. The Journal of Educational Research, 98(3), 164-175. doi:10.3200/joer.98.3.164175

Epstein, J. L. (2011). School, family and community partnerships. preparing educators and improving schools. Philadelphia, PA: Westview Press.

Escudero, J. M. (2005). Fracaso escolar, exclusión educativa: ¿de qué se excluye y cómo? Profesorado. Revista de Curriculum y Formación del Profesorado, 9(1), 1-24

Escudero, J. M., González, M. T. y Martínez, B. (2009). El fracaso escolar como exclusión educativa: comprensión, políticas y prácticas. Revista Iberoamericana de Educación, 50, 41-64.

Fan, X. y Chen, M. (2001). Parental involvement and student's academic achievement: A metaanalysis. Educational Psychology Review, 13(1), 1-22.

Fernández, M., Mena, L. y Riviere, J. (2010). Fracaso y abandono escolar en España. Barcelona: Fundación La Caixa.

Fernández, M. (2005). Evaluación y cambio educativo: el fracaso escolar. Madrid: Morata.

Fullana, J. (1998). La búsqueda de factores protectores del fracaso escolar en niños en situación de riesgo mediante un estudio de casos. Revista de Investigación Educativa, 16(1), 47-70.

García, J. y Delgado, F. (1994). El rendimiento escolar. Los alumnos y las alumnas ante su éxito o fracaso. Madrid: Editorial Popular.

García, F. J. (1996). La participación de las familias en la educación de los hijos. En R. A. Clemente y C. Hernández (Comps.), Contextos de desarrollo psicológico y educación (pp. 257-282). Málaga: Aljibe.

Gil, P. (1999). Algunas aclaraciones actuales sobre el fracaso escolar. Recuperado de http://bit.ly/ibNvtr

Gimeno, J. (2005). La educación secundaria obligatoria: su sentido educativo y social. Madrid: Morata.

González, I. (2001). ¿Fracaso escolar o fracaso de la oferta y práctica educativas? Revista de Estudios de Juventud, 52, 51-58.

González, M. T. (2006). Absentismo y abandono escolar: una situación singular de exclusión educativa. REICE. Revista Iberoamericana sobre Calidad, Eficacia y Cambio en Educación, 4(1), $1-15$.

González-Pienda, J. A. y Núñez, J. C. (2005). La implicación de los padres y su incidencia en el rendimiento de los hijos. Revista de Psicología y Educación, 1(1), 115-134. 
Grau, R., Pina, T. y Sancho, C. (2011). Posibles causas del fracaso escolar y el retorno al sistema educativo. Hekadamos, Revista educativa digital, 9, 55-76.

Herrero, M. (2001). ¿Fracaso escolar o fracaso del sistema escolar? Revista de Estudios de Juventud, 2, 59-63.

Instituto Nacional de Estadística. (2015). Mujeres y hombres en España. Madrid: INE.

Instituto Nacional de Evaluación Educativa. (2013). Objetivos educativos europeos y españoles. Estrategia educación y formación 2020. Informe español 2013. Madrid: Secretaría de Estado de Educación.

López, N. (2009). Las metas educativas ante el nuevo panorama social y cultural de América Latina. En A. Marchesi, J. C. Tedesco y C. Coll (Coords.), Calidad, equidad y reformas en la enseñanza (pp. 35-46). Madrid: OEI.

López, M. T. y Martín Rasines, E. (2008). Familia y educación en valores. En, M. T. López (Aut.), Familia, escuela y sociedad. Responsabilidades compartidas en la educación (pp.13-51). Madrid: Ediciones Cinca.

López, M. T., Utrilla, A. y Valiño, A. (2006). Políticas públicas y familia. Análisis de la situación en España. Madrid: Ediciones Cinca.

Lozano, A. (2003). Factores personales, familiares y académicos que afectan al fracaso escolar en la Educación Secundaria. Revista Electrónica de Intervención Psicoeducativa y Psicopedagógica, 1(1), 43-66.

Marchesi, A. (2000). Controversias en la educación española. Madrid: Alianza Editorial.

Marchesi, A. (2003). El fracaso escolar en España. Madrid: Fundación Alternativas.

Marchesi, A. (2009). Las metas educativas 2021. Un proyecto iberoamericano para transformar la educación en la década de los bicentenarios. Revista Iberoamericana de Ciencia, Tecnología y Sociedad, 4(12), 87-157.

Marchesi, A. (2012). El Bienestar de los docentes en tiempos de crisis. Revista de la Facultad de Ciencias de la Educación, 12, 9-12.

Marchesi, A. y Martín, E. (Comps.) (2002). Evaluación de la educación secundaria. Fotografía de una etapa polémica. Madrid: Editorial SM.

Marchesi, A., Martínez, R. y Martín, E. (2004). Estudio longitudinal sobre la influencia del nivel sociocultural en el aprendizaje de los alumnos de educación secundaria obligatoria. Infancia y Aprendizaje, 27(3), 307-323. doi:10.1174/0210370042250059

Marchesi, A. y Pérez, E. (2003). La comprensión del fracaso escolar. En A. Marchesi y C. Hernández (Coords.), El fracaso escolar. Una perspectiva internacional (pp. 25-50). Madrid: Alianza Editorial.

Marina, J. A. (2010). Las culturas fracasadas. Barcelona: Anagrama.

Martínez, R. A. y Álvarez, L. (2005). Fracaso y abandono escolar en educación secundaria obligatoria: implicación de la familia y los centros escolares. Aula Abierta, 85, 127-146.

Martínez, R. A. y Pérez, M. H. (2004). Evaluación e intervención educativa en el campo familiar. Revista Española de Orientación y Psicopedagogía, 15(1), 89-104.

Meil, G. (2006). Padres e hijos en la España actual. Barcelona: Fundación La Caixa.

Ministerio de Educación, Cultura y Deporte. (2014). Plan para la reducción del abandono educativo temprano. Madrid: MECD. 
Cooperación entre las Familias y los Centros Escolares como Medida Preventiva...

L. Álvarez y R. A. Martínez González

Mora, A. J. (2010). Determinantes del abandono escolar en Cataluña: más allá del nivel socioeconómico de las familias. Revista de Educación, Num. Extraordinario, 171-190.

Moreno, T. (2010). La relación familia-escuela en secundaria: algunas razones del fracaso escolar. Profesorado. Revista del Curriculum y Formación del Profesorado, 14(2), 240-255.

Murillo, F. J. (2005). La investigación sobre eficacia escolar. Barcelona: Octaedro.

Murillo, F. J. (Coord.). (2007). Investigación iberoamericana sobre eficacia escolar. Bogotá: CAB.

Murillo, F. J. y Duk, C. (2011). ¿Escuelas eficaces versus escuelas inclusivas? Revista Latinoamerica de Educación Inclusiva, 5(1), 11-12.

Musitu, G. y Cava, M. J. (2001). La familia y la educación. Barcelona: Octaedro.

Musitu, G., Estévez, E. y Jiménez, T. (2010). Funcionamiento familiar, convivencia y ajuste en hijos adolescentes. Madrid: Ed. Cinca.

Navarrete, L. (2007). Jóvenes y fracaso escolar en España. Madrid: Instituto de la Juventud.

Navarro, N. (2001). La falta de apego hacia y desde la escuela. Revista de Estudios de Juventud, 52, $45-50$.

Pedró, F. (2012). Políticas públicas sobre apoyo y refuerzo educativo: evidencias internacionales. Revista de Educación, Num. extraordinario, 22-45.

Pérez de Pablos, S. (2003). El papel de los padres en el éxito escolar de los hijos. Madrid: Santillana.

Pérez-Díaz, V., Rodríguez, J. C. y Fernández, J. J. (2009). Educación y familia. Papeles de Economía Española, 119, 36-58.

Pérez-Díaz, V., Rodríguez, J. C. y Sánchez, L. (2001). La familia española ante la educación de los hijos. Barcelona: Fundación La Caixa.

Portillo-Torres, M. C. (2015). Propuesta de un nuevo enfoque para reducir el abandono escolar en secundaria. Revista Electrónica Educare, 19(2), 303-316. doi:10.15359/ree.19-2.17

Puig, J. M. (2003). Educación en valores y fracaso escolar. En A. Marchesi y C. Hernández, (Coords.). El fracaso escolar. Una perspectiva internacional (pp. 83-97). Madrid: Alianza Editorial.

Ramo, Z. (2000). Éxito y fracaso escolar. Culpables y víctimas. Barcelona: Cisspraxis.

Redding, S. (2006). The mega system: deciding, learning, connecting. Lincoln, IL: Academic Development Institute.

Robledo, P. y García, J. N. (2006). Factores psicológicos de los padres de alumnos con dificultades de aprendizaje. Revisión de estudios empíricos. En J. Uriarte y P. Martín (Eds.), Necesidades educativas especiales, contextos desfavorecidos y apoyo social (pp. 225-235). Bilbao: Psicoex.

Rodríguez-Martínez, C. y Blanco García, N. (2015). Diferencias de género, abandono escolar y continuidad en los estudios. Revista Iberoamericana de Educación, 68, 59-78.

Roldán, M. A. (2008). Familia y escuela como agentes de socialización. En T. López (Dir.), Familia, Escuela y Sociedad. Responsabilidades compartidas en la educación (pp. 95-142). Madrid: Ed. Cinca.

Rosario, P., Mourão, R., Núñez, J. C., González-Pienda, J. A. y Solano, P. (2006). Escuela-familia: ¿es posible una relación recíproca y positiva? Papeles del Psicólogo, 27(3), 171-179.

Sánchez, S. (2001). Niños y jóvenes en desventaja ante la educación: causas y consecuencias del absentismo y del fracaso escolar. Revista de Estudios de Juventud, 52, 23-26. 
Santín González, D. (2006). Familia, escuela y fracaso escolar. En M. T. López (Dir.), La familia en el proceso educativo (pp. 181-222). Madrid: Ed. Cinca.

Soler, P. (2003). Claves para reducir el fracaso escolar. En A. Marchesi y C. Hernández. (Coords.), El fracaso escolar. Una perspectiva internacional (pp. 287-301). Madrid: Alianza Editorial.

Suárez, N., Tuero-Herrero, E., Bernardo, A., Fernández, E., Cerezo, E., González-Pienda, J. A., ..., Núñez, J.C. (2011). El fracaso escolar en educación secundaria: análisis del papel de la implicación familiar. Revista de Formación del Profesorado e Investigación Educativa, 24, 49-64.

Symeou, L. (2006). Capital cultural y social: ¿qué podemos aprender para investigar y reforzar la colaboración entre la familia y la escuela. Cultura y Educación, 18(3-4), 219-229. doi:10.1174/113564006779173037

Symeou, L., Martínez-González, R. A. y Álvarez Blanco, L. (2012). Dropping out of high school in Cyprus: Do parents and the family matter? International Journal of Adolescence and Youth, 17(4) 1-19. doi:10.1080/02673843.2012.717899

Taborda, M. C., Formosinho, M. y Fonseca, A. C. (2007). Abandono escolar temprano. Datos de un estudio en la región centro de Portugal. Revista de Psicología y Educación, 1(1), 177-191.

Tarabini, A. y Curran, M. (2015). El efecto de la clase social en las decisiones educativas: un análisis de las oportunidades, creencias y deseos educativos de los jóvenes. Revista de Investigación en Educación, 13(1), 7-26

Torío, S., Hernández, J. y Peña, J. V. (2007). Capital social familiar y expectativas académicoformativas y laborales en el alumnado de educación secundaria obligatoria. Revista de Educación, 343, 559-586

Townsend, T. E. (2007). International handbook of school effectiveness and improvement. Dordrecht: Springer.

UNESCO. (2012a). Compendio mundial de educación. Oportunidades perdidas: el impacto de la repetición y de la salida prematura de la escuela. Montreal: Instituto de Estadística de la UNESCO.

UNICEF. (2012b). Completar la escuela. Un derecho para crecer, un deber para compartir. Panamá: UNICEF.

Valls, G. (2003). La transición de primaria a secundaria. Cuadernos de Pedagogía, 327, 64-66.

Vaquero, A. (2005). El abandono escolar temprano en España: programas y acciones para su reducción. Revista Galega do Ensino, 47, 1443-1464.

Wikeley, F., Stoll, L. y Lodge, C. (2002). Effective school improvement. Educational Research and Evaluation, 4, 363-385.

Zelmanovich, P. y Levinsky, R. (2012). Socialización y deseo de aprender: Brújulas para fortalecer la trayectoria escolar. Revista Latinoamericana de Inclusión Educativa, 6(2), 111-136. 


\section{Breve CV de las autoras}

\section{Raquel-Amaya Martínez-González}

Catedrática de Universidad Acreditada (ANECA) y Profesora Titular de Universidad en el Departamento de Ciencias de la Educación de la Universidad de Oviedo (España). Coordina el Grupo de Investigación "Intervención Educativa en el ámbito Familiar, Escolar y Social” (IEFES) y desarrolla estudios y programas educativos con Familias y Profesionales del sector Educativo, Servicios Sociales, de la Salud y otros sectores afines. Ha recibido Premios Nacionales en España por sus trabajos sobre Familia y Educación. Ha presidido el European Research Network About Parents in Education (ERNAPE). Es miembro del International Network of Scholars on School-Family-Community Partnerships (INET), USA. Es Convenor de la European Educational Research Association en el Network on Communities, Families, and Schooling in Educational Research. Ha difundido sus resultados de investigación en revistas y conferencias nacionales e internacionales. Email: raquelamaya@gmail.com

\section{Lucía Álvarez Blanco}

Doctora en Pedagogía y Profesora Ayudante Doctora acreditada -ANECA-, Maestra, Mediadora Familiar y Profesora en el Departamento de Ciencias de la Educación de la Universidad de Oviedo. Como miembro del Grupo de Investigación "Intervención Educativa en el ámbito Familiar, Escolar y Social (IEFES)” colabora desarrollando investigaciones y programas socioemocionales y educativos con familias, centros escolares y profesionales. Es miembro de varios proyectos internacionales y nacionales, presentando los resultados de estas investigaciones en congresos y revistas de reconocido prestigio. Es Convenor de la European Educational Research Association en el Network on Communities, Families, and Schooling in Educational Research. Email: alvarezblucia@uniovi.es 